\section{European heavens}

\section{Robert Connon Smith}

Fundamental Astronomy. Edited by H. Karttunen, P. Kröger, H. Oja, M. Poutanen and K.J. Donner. SpringerVerlag:1987. Pp.478. DM 128, £47, \$45.

ONE OF the important differences between North American and European universities is that in the United States and Canada the great majority of students studying astronomy are liberal arts majors with little or no command of mathematics. The descriptive nature of textbooks for these courses is well known; there are many excellent examples, but they are not very suitable for the typical European student of astronomy, who has a training in basic mathematics and physics. For such students, there is a variety of advanced texts but a dearth of elementary accounts that include mathematics.

Fundamental Astronomy goes a long way towards filling that gap. The topics covered are fairly standard for an introductory text, ranging from spherical astronomy to cosmology, via instruments, planets, stars and galaxies. However, the balance is better than usual. Instead of the stress on the Solar System that is common in descriptive texts, space is given to more mathematical topics, such as radiation mechanisms and celestial mechanics, with a brief but useful chapter explaining the different ways of defining temperature.

Unusually, the book has a large number of authors. It evolved from lecture notes by one of the editors, but has grown with the help of no fewer than 13 colleagues other than the five editors. This wide range of expertise gives the book an authority that would be almost impossible for a single-author text. The translation from the original Finnish is excellent.

Athough the treatment makes integral use of mathematics, much of the book would be accessible to someone who has followed that subject to an intermediate level at secondary school. As a result, Fundamental Astronomy would also be a good choice for the serious amateur who wants more than just description but whose mathematics cannot cope with more advanced texts. As the editors point out, the advent of personal computers has led to a need for amateurs to have access to exact, but comprehensible, mathematical formalism for their programs.

There is a series of helpful appendices, ranging from elementary calculus to the Lorentz transformation, and including 24 tables of basic information. Some of these are the standard numerical values of physical constants (in SI units); others are more unusual, such as the lists of large telescopes (optical, radio and millimetre). In the text itself, there are other aids to the reader: worked examples are provided at the end of more than half the chapters, while starred sections in small print take the inquisitive reader beyond the general level of the book. Finally, the volume is enhanced by its illustrations, in particular by the central colour supplement.

Robert Connon Smith is a Lecturer in the Astronomy Centre, University of Sussex, Falmer, Brighton BN1 $9 Q H, U K$.

\section{Stars in a jar}

\section{Grenville Turner}

Chemistry of the Solar System: An Elementary Introduction to Cosmochemistry. By Hans E. Suess. Wiley: 1987. Pp. 143. $£ 21.50, \$ 24.95$.

Cosmochemistry is the subject that brings astronomy and astrophysics into the laboratory. By studying the chemical, isotopic and mineralogical make-up of primitive meteorites, cosmochemists have been able to provide evidence on which to base theories of the formation of the elements in stellar interiors, as well as theories of the formation of the Solar System from the debris of these stellar furnaces. Professor Hans Suess has been a major contributor to the subject for over half a century, so the publication of an introductory text by him is a significant event. The book is based on a course of lectures given from 1968 to 1985 at the University of California, San Diego.

The text is divided into two parts. The first covers the nuclear physics related to elemental and isotope abundances and element synthesis, while the second concentrates on various aspects of the chemistry of the early Solar System, deduced principally from meteorite studies, though there is also a brief discussion of the planets, asteroids and comets. The content is somewhat unusual and clearly reflects an unashamed bias towards those aspects on which the author has worked. Professor Suess has had a hand in so many areas that this approach gives a reasonable covbeen better, and as the end of the book approaches subjects are covered with increasing speed and decreasing depth.

The bulk of the first part is given over to a detailed discussion of nuclear stability, shell structure and element abundance systematics, all areas in which Professor Suess was a leading figure in the 1950 s. The treatment of mechanisms of nucleosynthesis is in contrast perfunctory, and is largely restricted to an elementary discussion of the $\mathrm{r}$ - and s-processes. In view of the current flood of evidence for pre-solar isotopic anomalies in meteorites, assesserage, although the balance could have
ADVERTISEMENT

\section{Journals}

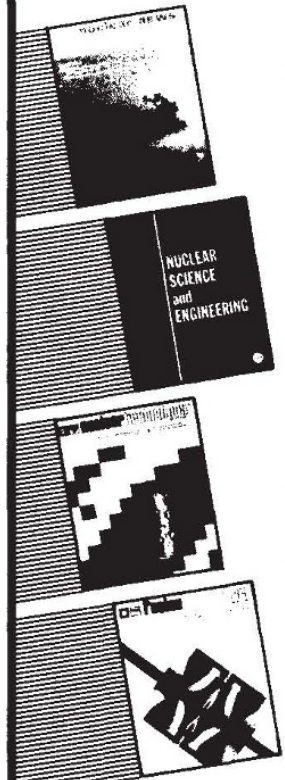

Your number one source for current information about NUCLEAR ENERGY

NUCLEAR NEWS

NUCLEAR SCIENCE \& ENGINEERING NUCLEAR TECHNOLOGY

TRANSACTIONS

REMOTE SYSTEMS TECHNOLOGY PROCEEDINGS

FUSION

TECHNOLOGY

For free catalog

AMERICAN NUCLEAR SOCIETY 555 N. Kensington Avenue

La Grange Park, Illinois 60525 USA 312-352-6611 Telex: 4972673

Reader Service No.30

ment of the implications for other nucleosynthetic processes is noticeably absent. The account of isotope anomalies is itself very patchy with several pages given over to the noble gases and virtually nothing to the so-called FUN anomalies in elements such as calcium and titanium.

The weakness of the book is that it ignores some of the more recent advances in the subject. Some of these omissions, while arguably minor in the overall context, are particularly irritating. The discussion of sources of ${ }^{3} \mathrm{He}$ in the Earth's atmosphere, for example, ignores the observation made in the author's own institute that (primordial) ${ }^{3} \mathrm{He}$ is being released into the oceans by volcanic activity at the mid-ocean ridges.

The omission of recent work is perhaps not altogether a bad feature, in that the real strength of book is the way it provides a perspective on cosmochemistry covering the past 50 years (half of the references are to papers published before 1954). In case my review seems too negative, let me conclude by saying that the book is well written and, apart from a few minor errors, enjoyable to read. Combined with one of the modern texts on meteorites, it is a good introduction to an exciting and rapidly moving subject.

Grenville Turner is a Professor in the Department of Physics, University of Sheffield, Sheffield S3 $7 R H, U K$ 